\title{
Cycle biologique du Nématode Rictulaire Pterygodermatites (Multipectines) affinis (Jagerskiold, 1904)
}

\author{
par J.-C. QUENTIN *, C. SEUREAU ** et R. VERNET *** \\ * Laboratoire de Zoologie (Vers) associé au C.N.R.S., Muséum national d'Histoire naturelle, \\ 43, rue Cuvier, F 75231 Paris Cedex 05. \\ ** Laboratoire d'Histophysiologie des Insectes ( $\mathrm{P}^{\mathrm{r}} \mathrm{R}$. MarToJa), \\ Université Pierre-et-Marie-Curie, 12, rue Cuvier, F 75005 Paris. \\ *** Centre de Recherche sur les zones arides, Benis-Addès, Saoura, Algérie.
}

\section{Résumée.}

Des larves infestantes du Nématode Rictulariidae Pterygodermatites affinis (Jägerskiold, 1904) sont identifiées chez des Pimélies Tachyderma hispida (Forskael) d'Algérie.

L'infestation d'un jeune chat permet la récolte d'œufs embryonnés après 38 jours de développement et la récolte d'un parasite femelle.

Le cycle biologique expérimental de ce Rictulaire chez Locusta migratoria se caractérise par la brièveté du développement et par une croissance comparable au cours du premier et du deuxième stade larvaires.

La réaction cellulaire de l'Insecte aux larves de $P$. affinis est plus faible chez la Pimélie que chez le Criquet hôte expérimental.

\section{Summary:}

Biological cycle of the Nematode Rictulariidae : Pterygodermatites (Multipectines) affinis (Jägerskiold, 1904).

Infestant larva of the Nematode Rictulariidae: Pterygodermatites affinis (Jägerskiold, 1904) are localized in Pimelia: Tachyderma hispida Forskael of Algeria.

Through the infestation of a young cat, embryonated eggs are collected after 38 days of development altogether with a female parasite. 
The experimental cycle of this rictulariid nematode in Locusta migratoria is characterized by the shortness of its development and the similarity in growth during the first and second larval stages.

The cellular reaction of the Insect to the larva $P$. affinis is weaker in the Pimelia than in the Grasshopper, used as experimental host.

\section{Introduction}

Les Pimélies d'Afrique du Nord sont fréquemment infestées par des larves de Nématodes hétéroxènes qui évoluent spontanément chez ces hôtes intermédiaires. Parmi les différentes espèces rencontrées, un petit nombre de larves infestantes peuvent être aisément identifiées, car leurs structures céphaliques sont comparables à celles de l'adulte correspondant. C'est le cas, notamment, des espèces Spirura rytipleurites seurati Chabaud, 1954 et Streptopharagus kutassi Schulz, 1927.

Cependant, dans leur majorité, les larves infestantes récoltées ont des structures céphaliques qui diffèrent profondément de celles de l'aduilte. L'identification de la larve peut alors être orientée en comparant sa morphologie à celle de larves de Nématodes du même groupe systématique, qui sont déjà connues.

Une telle comparaison, fondée, d'une part; sur la localisation de la larve chez l'Insecte, et, d'autre part, sur ses structures céphaliques, cuticulaires et anatomiques, nous a ainsi permis de classer parmi les Nématodes Rictulariidae des larves infestantes récoltées chez des Pimélies Tachyderma hispida (Forskael) rapportées en septembre 1974 de Beni-Abbès.

Ces larves présentent en effet les caractères suivants: elles occupent chez la Pimélie une localisation intra-épithéliale extrêmement précise dans la paroi de l'intestin postérieur en arrière des tubes de Malpighi; elles sont courtes et trapues, leur corps est courbé et contracté sur la face dorsale ; elles sont munies d'une capsule buccale déjà différenciée, deux ailes latérales striées transversalement ornent les faces latérales.

Par ailleurs, nous avons été guidés, pour l'identification spécifique de ces larves et dans le choix d'un hôte définitif susceptible d'être infesté, par un ensemble de caractères très spécialisés :

- l'œsophage n'a pas la forme en massue communément observée chez les Rictulaires, mais celle de Spirurides évolués ; il se compose en effet d'une portion musculaire réduite, nettement individualisée d'une portion glandulaire massive ;

- l'extrémité caudale n'est pas inerme comme celle des autres troisièmes stades de Rictulaires, mais ornée de minuscules épines disposées en séries linéaires;

- enfin, la tête porte un cadre buccal évasé en collerette sur les faces dorsale et ventrale et se soulevant latéralement en deux pointes. Ces pointes cuticulaires sont également observées chez les larves de Pterygodermatites taterilli (Baylis, 1928) (Cf. Quentin, 1970), qui est une espèce dont l'adulte a des structures céphaliques comparables à celles de $P$. affinis (Jägerskiold, 1904). 

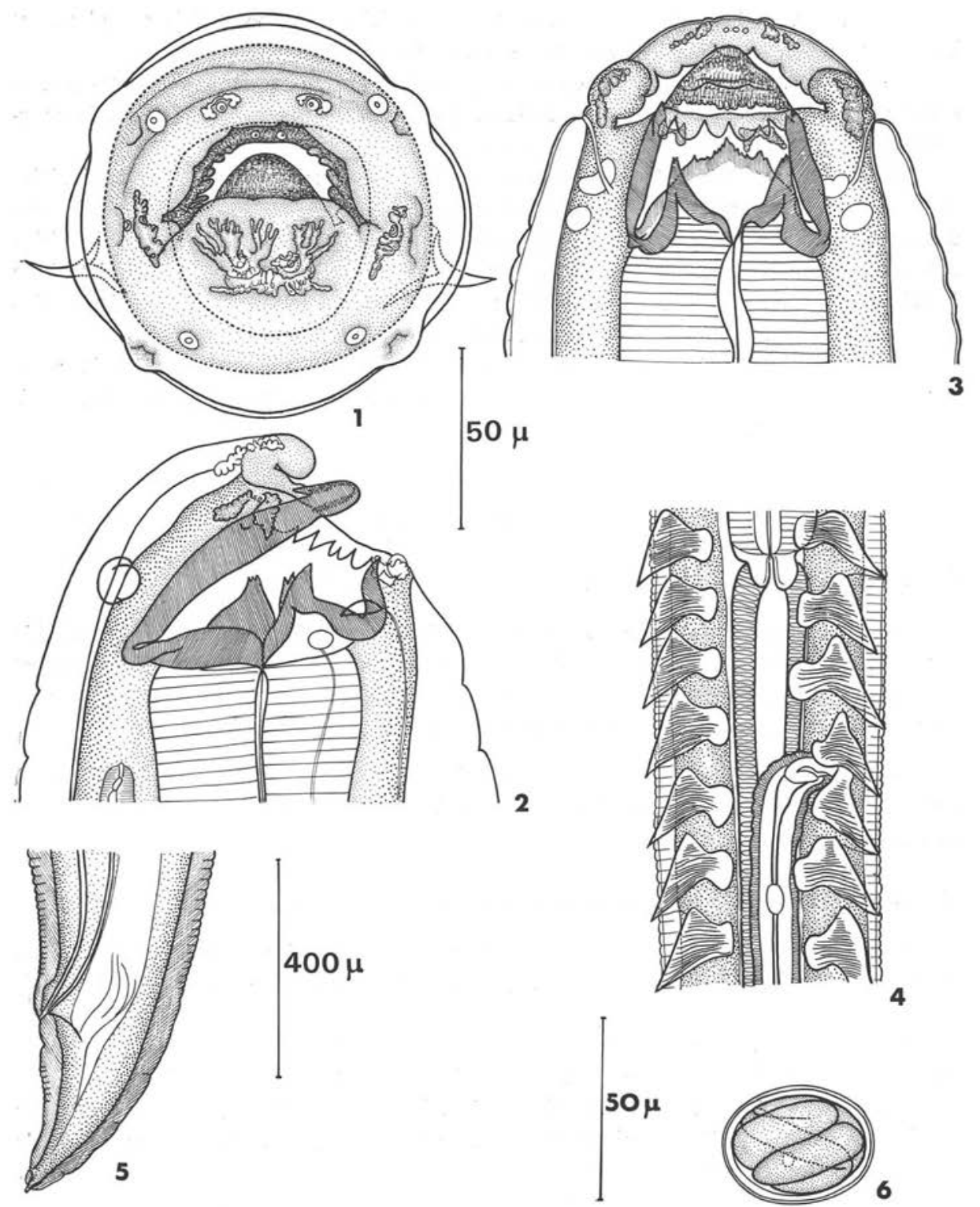

4

PL. I. - Jeune femelle de Pterygodermatites affinis (Jägerskiold, 1904) âgée de 53 jours.

Fig. 1, 2, 3. - Tête de cette femelle représentée successivement en vues apicale, dorsale .et latérale gauche. Fig. 4. - Détail de l'ornementation cuticulaire du corps au niveau de la vulve. Fig. 5. - Extrémité caudale du corps. Fig. 6. - Euf.

Fig. 1, 2, 3 : éch. $50 \mu$; Fig. 4, 5 : éch. $400 \mu$. 
Les structures larvaires de ce Nématode Rictulaire sont donc déjà complexes et indiquent qu'il s'agit d'une espèce très évoluée dans le groupe.

Nous avons donc pensé que ces larves pouvaient appartenir à l'espèce Pterygodermatites (Multipectines) affinis, dont l'adulte, parasite de Carnivores, présente la morphologie la plus différenciée.

Effectivement, l'infestation d'un jeune chat, né à Paris, avec 9 larves récoltées chez la Pimélie, permettait l'obtention d'œufs embryonnés dans les fèces après une: durée de 38 jours, correspondant à la période constatée entre l'infestation de l'hôte définitif et l'émission des œufs chez les Nématodes Rictulaires; en outre, le rejet d'une femelle gravide de $P$. affinis ( $P l$. I), 53 jours après l'infestation du chat, confirma définitivement notre hypothèse, sans qu'il ait été nécessaire de sacrifier le chat.

Nous complétons l'étude du développement larvaire de ce Rictulaire chez l'Insectepar l'infestation expérimentale d'Orthoptères Locusta migratoria L. avec des œufs. embryonnés du parasite.

\section{Cycle biologique}

\section{I. - Allure du développement.}

- Chronologie des mues. Le cycle biologique de ce Rictulaire est remarquablepar la brièveté de son développement. A la température de $28^{\circ} \mathrm{C}$, les larves émigrent dans la valvule proctodéale quelques heures après l'infestation, effectuent leur première mue du $2^{\circ}$ au $3^{e}$ jour et deviennent infestantes du $5^{\circ}$ au $7^{\circ}$ jour.

- Croissance larvaire. Les larves ont à l'éclosion une longueur inférieure à$300 \mu$, elles mesurent $505 \mu$ au début du deuxième stade, et atteignent $725 \mu$ à $780 \mu$. au troisième stade.

\section{II. - Morphologie et organogénèse des stades larvaires.}

Premier Stade. A l'éclosion, la larve porte dans sa région apicale et sur la facelatérale gauche un petit crochet, l'extrémité caudale est ornée de minuscules aspérités: cuticulaires.

Après 18 heures de développement chez l'Insecte, les caractères anatomiques decette larve se précisent en une ébauche œsophagienne et une ébauche intestinale très fines, en une cellule excrétrice allongée et plaquée contre l'isthme œsophagien, en une cellule R1 située au 1/3 postérieur de l'ébauche intestinale, et en un rectum formé detrois cellules regroupées.

Cette larve mesure $300 \mu$ de long sur $11 \mu$ de large et les dimensions de ses ébauches. sont les suivantes : œsophage $144 \mu$, intestin $96 \mu$, rectum $15 \mu$, pore excréteur situé à $108 \mu$ de l'apex, cellule R 1 située à $40 \mu$ en avant du pore anal, longueur de la queue 48-54 $\mu$.

L'organogénèse est très rapide, car, après $48 \mathrm{~h}$ de développement ( $P l$. II, fig. 3), la cellule R1 a donné naissance à quatre files cellulaires de 6 à 8 cellules chacune, 

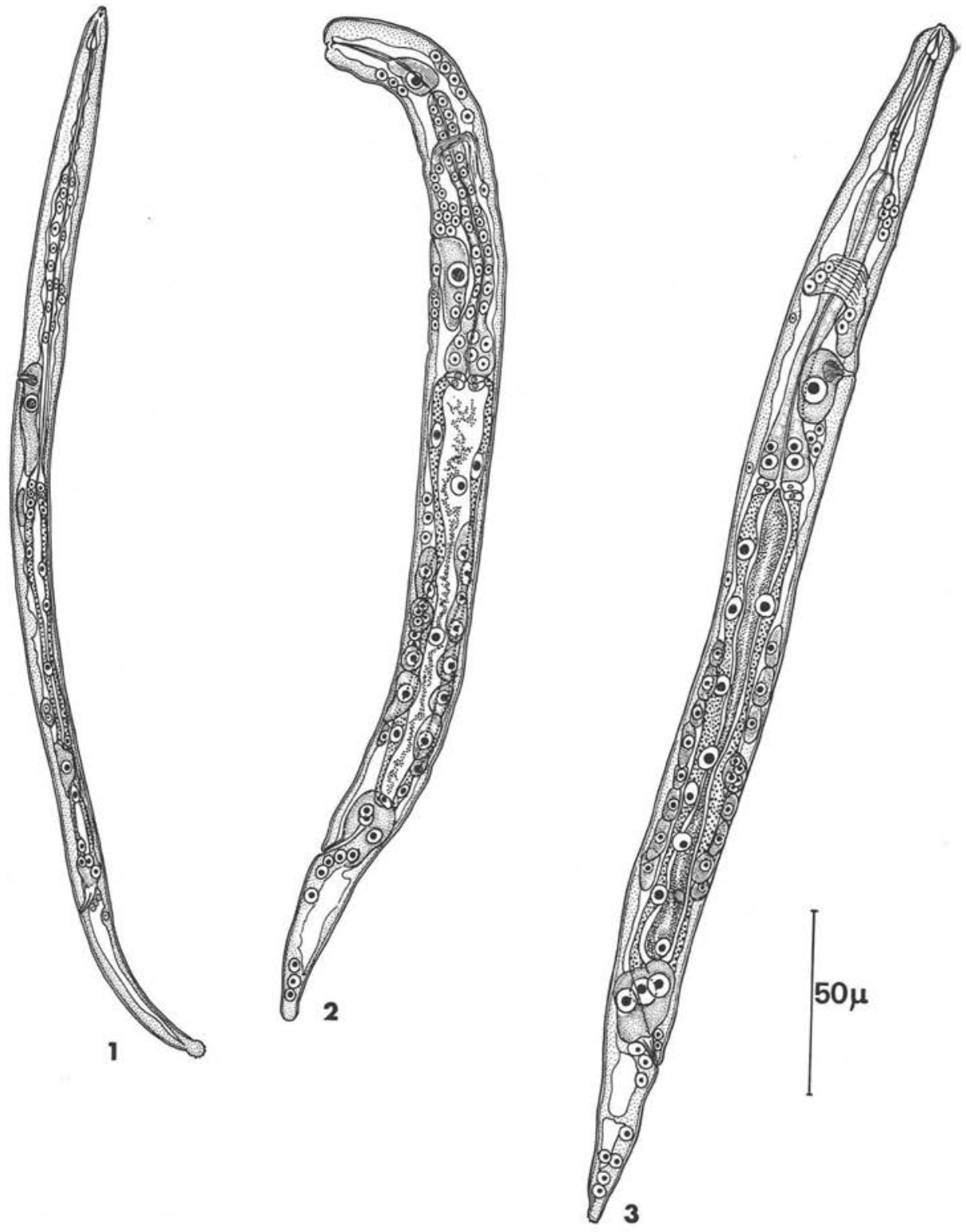

PL. II. - Pterygodermatites affinis (Jägerskiold, 1904). Premier stade larvaire.

Fig. 1. - Larve âgée de 18 h. Fig. 2. - Larve âgée de 36 h. Fig. 3. - Larve âgée de 48 h. Fig. 1, 2, 3 : éch. $50 \mu$. 
recouvrant latéralement l'ébauche génitale de quatre noyaux et enserrant la partie postérieure de l'intestin. Huit à neuf noyaux des cellules de la paroi intestinale sont alors comptés. Le rectum devient plus volumineux.

Ces larves mesurent de 315 à $350 \mu$ de long. Une larve longue de $350 \mu$ présente les dimensions suivantes: longueur $350 \mu$, largueur $42 \mu$, anneau nerveux et pore excréteur situés à $75 \mu$ et $97 \mu$ de l'apex, ébauches œsophagienne et intestinale de même taille: $140 \mu$, longueur du rectum $30 \mu$, ébauche génitale située à $90 \mu$ de l'anus, longueur de la queue $48 \mu$.

DeuXIÈme stade. Après $66 \mathrm{~h}$ de développement, la larve est déjà au deuxième stade (Pl. III, fig. 1). La principale transformation réside dans l'apparition d'une capsule buccale, qui communique, après un segment cuticulaire très court, avec l'œsophage. Ce dernier s'est particulièrement développé dans la région antérieure du corps -et a perdu sa forme rhabditoïde; le nombre des cellules intestinales s'est également accru et atteint environ 14 cellules. Les files cellulaires issues de la division de la cellule R1 sont incorporées à la paroi du corps.

Les dimensions de cette larve, au début du deuxième stade, sont les suivantes: longueur $505 \mu$, largeur $25 \mu$, anneau nerveux et pore excréteur situés respectivement à $70 \mu$ et $100 \mu$ de l'apex, capsule buccale profonde de $9 \mu$.

La croissance du Nématode se poursuit rapidement au deuxième stade, marquée principalement par le développement de l'intestin, où se condensent les restes diffus -du corps interne, qui occupait la lumière intestinale au cours du premier stade. La larve -atteint $550 \mu$ de long après trois jours et $725 \mu$ après quatre jours de développement (Pl. III. fig. 3). La larve est alors à la fin du $2^{\circ}$ stade larvaire, ainsi qu'en témoigne un léger décollement de l'exuvie dans la région caudale, et l'apparition des structures pharyngiennes du $3^{\mathrm{e}}$ stade (Pl. III, fig. 4).

Les dimensions de cette larve sont les suivantes: longueur $725 \mu$, largeur $36 \mu$, anneau nerveux et pore excréteur situés respectivement à $80 \mu$ et $112 \mu$ de lapex, profondeur de la -capsule buccale de $15 \mu$, longueur de l'œsophage $238 \mu$, longueur de l'intestin $375 \mu$, ébauche génitale située à $290 \mu$ en avant de l'anus, longueur du rectum $50 \mu$, longueur de la queue $55 \mu$.

Troisième stade (Pl. IV). De profondes modifications séparent cette larve du $2^{\circ}$ stade larvaire; elles concernent essentiellement les structures céphaliques, le tractus digestif, la position relative de l'ébauche génitale et l'ornementation cuticulaire de Ta larve.

Structures céphaliques. Leur aspect est tout-à-fait original par la différenciation des structures buccales et péribuccales ( $P l$. IV , fig. 1, 2, 3, 4, 5).

$\mathrm{La}$ bouche est garnie de trois dents pharyngiennes dont, seule, la dorsale est denticulée. Elle est entourée d'un cadre buccal chitinoïde de forme hexagonale, mesurant $8 \mu$ dans le sens dorso-ventral et $6 \mu$ dans le sens latéral ; la paroi externe incorpore en partie les 6 papilles labiales externes. En arrière de chaque papille latérale du cycle interne, le cadre se soulève pour former deux pointes aiguës. Le cadre se dilate en une 


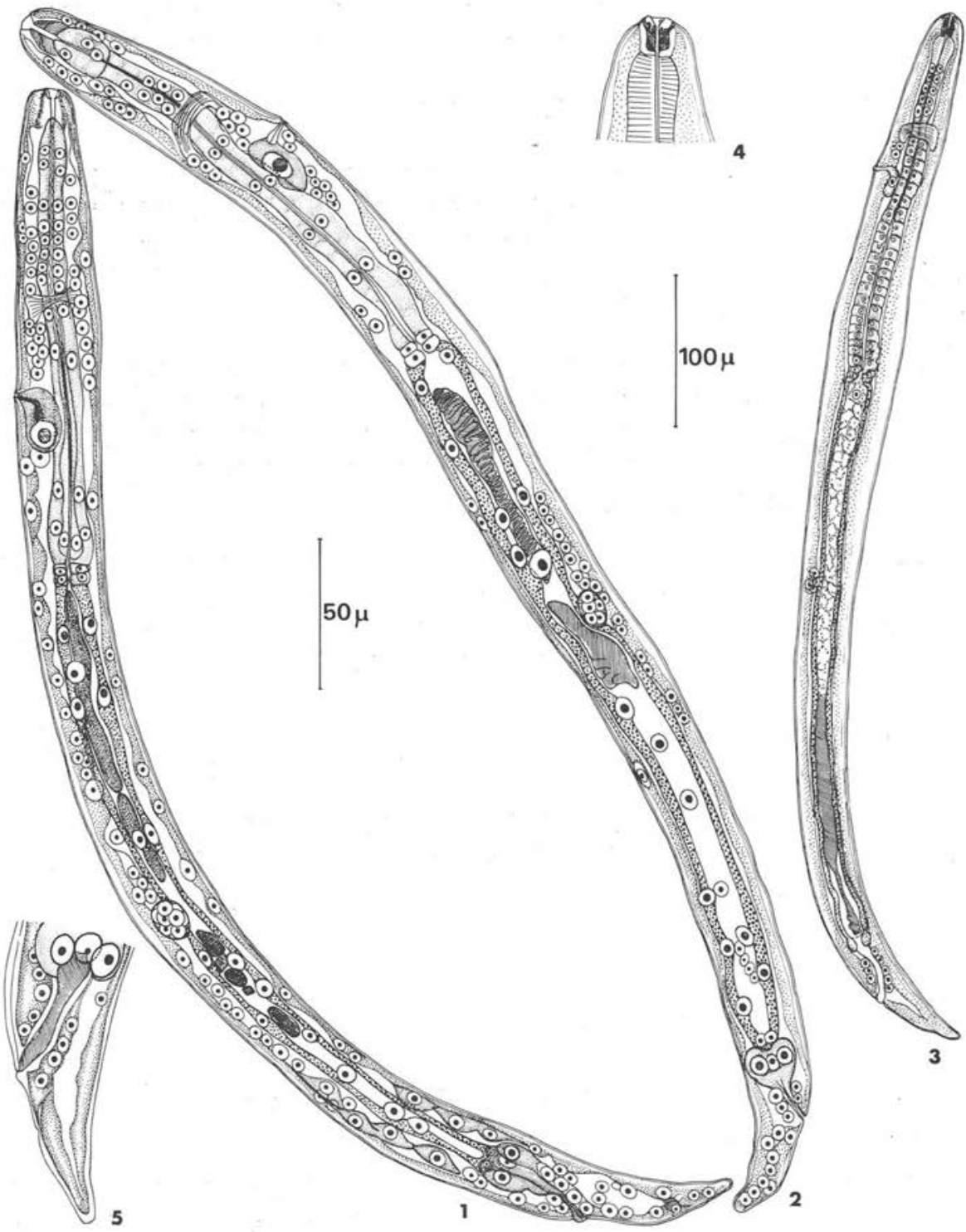

PL. III. - Pterygodermatites affinis (Jägerskiold, 1904). Deuxième stade larvaire.

Fig. 1. - Larve âgée de 66 h. Fig. 2. - Larve âgée de 3 jours. - Fig. 3. - Larve âgée de 4 jours. Fig. 4. - Détail de l'extrémité céphalique à la fin du deuxième stade, on remarque l'apparition des structures pharyngiennes du troisième stade. Fig. 5. - Décollement de l'exuvie dans la région caudale.

Fig. 1, 2, 4, 5 : éch. $50 \mu$; Fig. 3 : éch. $100 \mu$. 
fine collerette sur les faces dorsale et ventrales échancrées au niveau des amphides. La tête porte en outre quatre papilles labiales externes et quatre papilles céphaliques.

- Tractus digestif. Alors que le corps de la larve est, à la fin du deuxième stade larvaire, occupé principalement par l'intestin chargé de réserves, celui de la larve au troisième stade est caractérisé par la différenciation d'un volumineux œsophage glandulaire, qui repousse l'intestin dans la moitié postérieure du corps. Celui-ci, dont les dimensions se sont réduites, semble donc, chez la larve infestante, avoir perdu le rôle de réserve qu'il occupait au deuxième stade, au profit de l'œsophage glandulaire et de la paroi du corps, qui se sont épaissis.

- Position relative de l'ébauche génitale. L'ébauche génitale, située juste en avant de la cellule R1 sur la larve du premier stade, est encore, au deuxième stade, très en arrière (distance: $135 \mu$ ) de la jonction œsophage-intestin. Chez la larve infestante, ramassée sur elle-même, la distance séparant l'ébauche génitale de la jonction œsophage-intestin est réduite à $40 \mu$.

- Ornementation cuticulaire. Elle est constituée de deux épaisses ailes latérales qui parcourent le corps sur toute la longueur et s'arrêtent en arrière de l'anus, et par un bourgeon de fines épines cuticulaires réparties en plus grand nombre sur la face ventrale de l'extrémité caudale ( $P l . I V$, fig. 9, 10, 11).

Les longueurs des larves du troisième stade obtenues expérimentalement 5 à 7 jours après l'infestation de Locusta migratoria varient de 725 à $745 \mu$.

Les larves recueillies chez la Pimélie Tachyderma hispida sont légèrement plus grandes et atteignent $780 \mu$ de long; leurs autres dimensions sont les suivantes:

Largeur $50 \mu$; anneau nerveux, pore excréteur et deirides situés respectivement à $80 \mu$, $110 \mu$ et $130 \mu$ de l'apex; capsule buccale profonde de $11 \mu$; longueurs respectives des parties musculaire et glandulaire de l'œsophage de $100 \mu$ et $248 \mu$, longueur de lintestin $350 \mu$, ébauche génitale située à $420 \mu$ de l'apex, longueur de la queue $68 \mu$, épines caudales disposées sur une longueur de $13 \mu$.

\section{III. - Morphologie et dimensions de la femelle adulte obtenue expérimenta- lement.}

Cette femelle, obtenue après un développement de 53 jours chez le chat, possède 131 paires de peignes cuticulaires, dont 48 sont prévulvaires. Nous en détaillons sa morphologie sur la planche 1 .

Ses dimensions sont les suivantes: longueur du corps $2 \mathrm{~cm}$, largeur $440 \mu$, anneau nerveux, pore excréteur et deirides situés respectivement à $300 \mu, 450 \mu$ et $520 \mu$ de l'apex ; capsule buccale profonde de $47 \mu$; longueurs respectives de l'œsophage musculaire et de l'œsophage glandulaire 440 et $2950 \mu$; vulve située à $3600 \mu$ de l'apex; dimensions des œufs $44 \times 32 \mu$; longueur de la queue $240 \mu$. 

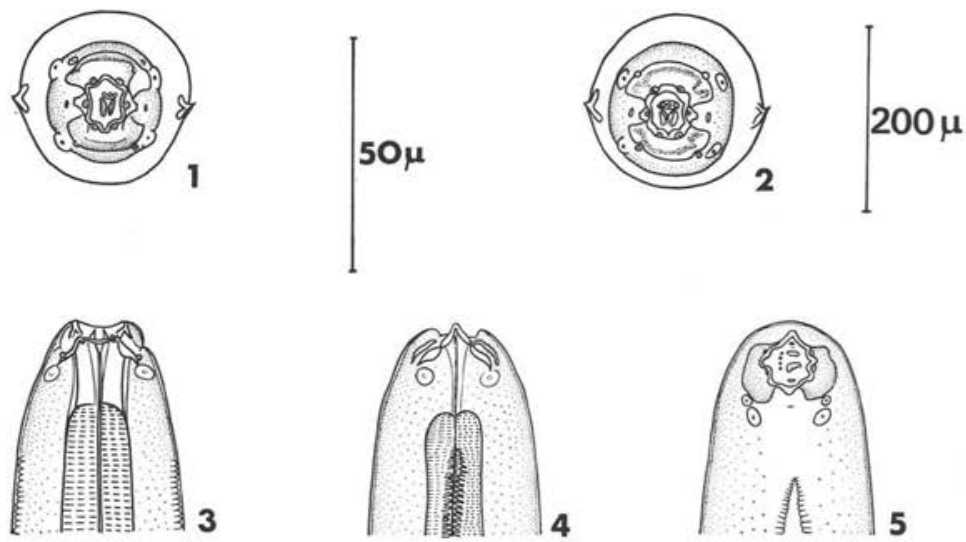

5

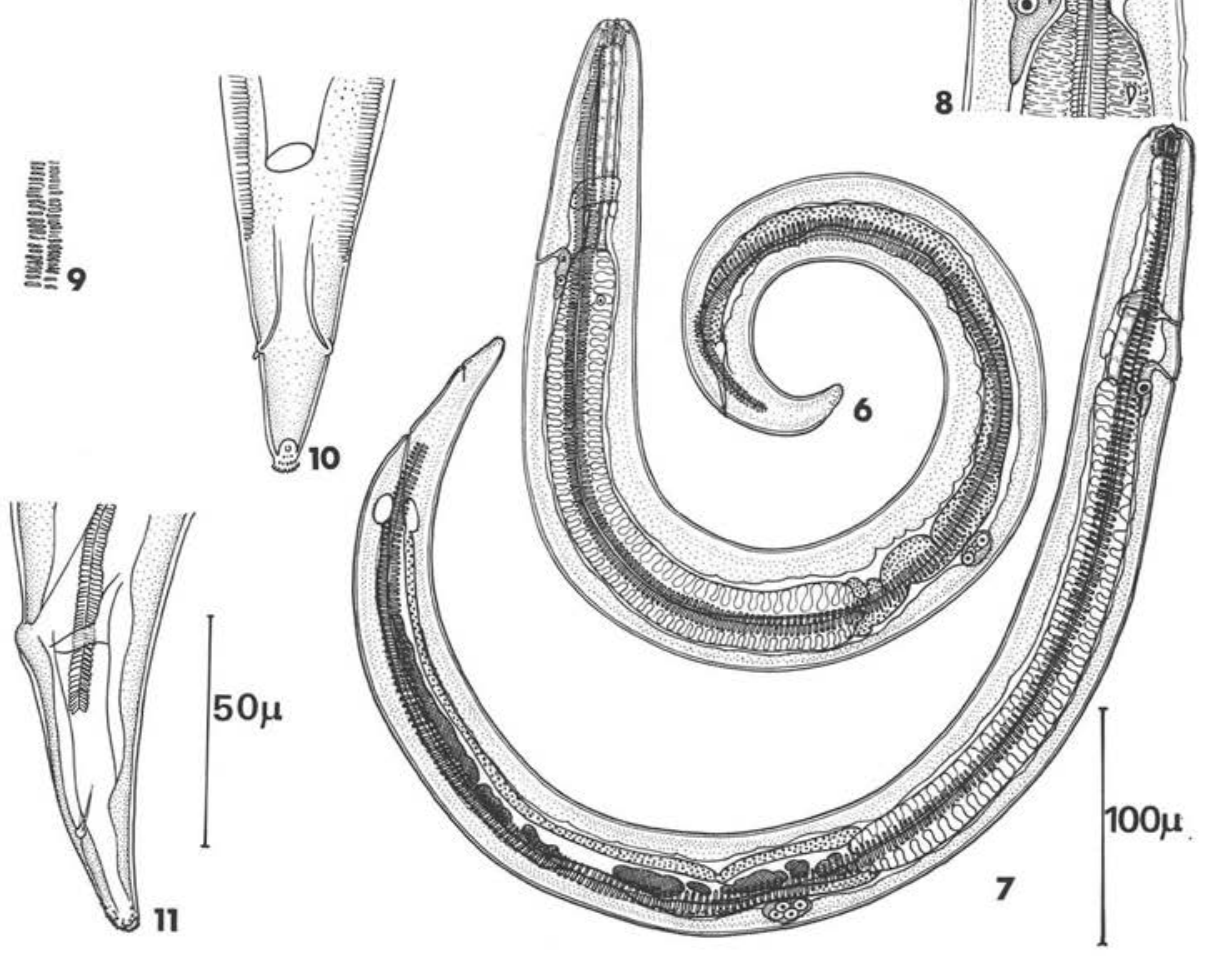

PL. IV. - Pterygodermatites affinis (Jägerskiold, 1904). Troisième stade larvaire.

Fig. 1, 2. - Têtes de deux larves en vues apicales. On remarque le cadre buccal hexagonal. Fig. 3, 4, 5. - Extrémité céphalique représentée successivement en vues ventrale, latérale et oblique. Fig. 6 et 7. - Larves infestantes au repos, représentées en vue latérale. Fig. 8. - Extrémité antérieure du corps montrant la différence d'épaisseur entre l'œsophage musculaire et l'œsophage glandulaire. Fig. 9. - Détail de l'aile cuticulaire latérale. Fig. 10 et 11. - Extrémité caudale du corps en vues ventrale et latérale.

Fig. $1,2,3,4,5,9,10,11$ : éch. $50 \mu$; Fig. 6 et 7 : éch. $100 \mu$; Fig. 8 : éch. $200 \mu$. 


\section{IV. - Réactions cellulaires chez l'Insecte.}

- Chez la Pimélie (hôte naturel). Les larves infestantes de P. affinis sont localisées dans la partie la plus antérieure de l'iléon de Tachyderma hispida, à la limite entre l'intestin moyen et l'intestin postérieur.

Elles sont totalement incluses dans l'épaisseur de la paroi du tube digestif, dont l'épithélium, à ce niveau, présente des replis amples et nombreux.

On ne note pas de réactions histopathologiques particulières de la part des tissus qui entourent les larves et qui, de ce fait, constituent une capsule d'un diamètre moyen de 100 à $150 \mu$, formée simplement de l'épithélium intestinal du côté de la lumière du tube digestif et de la basale de cet épithélium du côté de l'hémocèle. Cette basale semble d'ailleurs souvent rompue et la larve n'est alors isolée de l'hémocèle que par l'assise musculaire qui représente la couche externe de la paroi intestinale ( $P l . I V$, fig. 1).

- Chez le Criquet (hôte expérimental). Comme chez l'hôte naturel, les larves du Nématode sont encapsulées dans la partie antérieure de l'iléon de Locusta migratoria, au niveau de la vulve proctodéale, et dans l'épaisseur de la paroi du tube digestif.

Par contre, les parasites sont entourés d'une capsule provenant d'une réaction histopathologique de l'épithélium de l'iléon ( $P l . V$, fig. 2). la structure de la paroi de cette capsule et, plus particulièrement, les cytoplasmes hypertrophiés et la couche fibreuse interne, montrent qu'il s'agit d'une capsule caractéristique des Rictulariidae, après une infestation expérimentale de Criquets (Seureau, 1973). Le diamètre moyen de cette capsule est de 150 à $200 \mu$.

\section{Discussion}

Le cycle biologique du Nématode Spiruride Pterygodermatites multipectines affinis présente un triple intérêt : 1) dans la reconnaissance taxonomique des Rictulaires au niveau des stades larvaires, 2) dans l'écologie du parasite, 3) dans l'étude comparée des réactions cellulaires que provoquent, chez l'Insecte, les Nématodes Rictulariidae.

PL. V.- Encapsulement des larves de troisième stade chez l'Insecte - hôte intermédiaire.

FIG. 1. - Pterygodermatites affinis chez Tachyderma hispida, Coléoptère (hôte naturel). Remarquer l'absence de réaction histopathologique de l'iléon. Le parasite ne semble être isolé de l'hémocèle que par des fibres de la musculature péri-intestinale (Mu). Fig. 2. - B. affinis chez Locusta migrateria, Orthoptère (hôte expérimental). Une capsule épithéliale enveloppe les nématodes. L'ensemble est inclus dans l'épaisseur de la paroi de l'iléon. Fig. 3. $-P$. taterilli chez L. migratoria (hôte expérimental). Même type d'encapsulement que pour la Fig. 2. Fig. 4. $-P$. hispanica chez L. migratoria (hôte expérimental). Des nématodes et la capsule épithéliale qui les enveloppe sont libérées dans l'hémocèle.

$\mathrm{Ne}=$ Nématode $; \mathrm{Ca}=$ capsule $; \mathrm{Ei}=$ épithélium de l'iléon $; \mathrm{He}=$ Hémocèle ;

Trait d'échelle $=50 \mu$. 

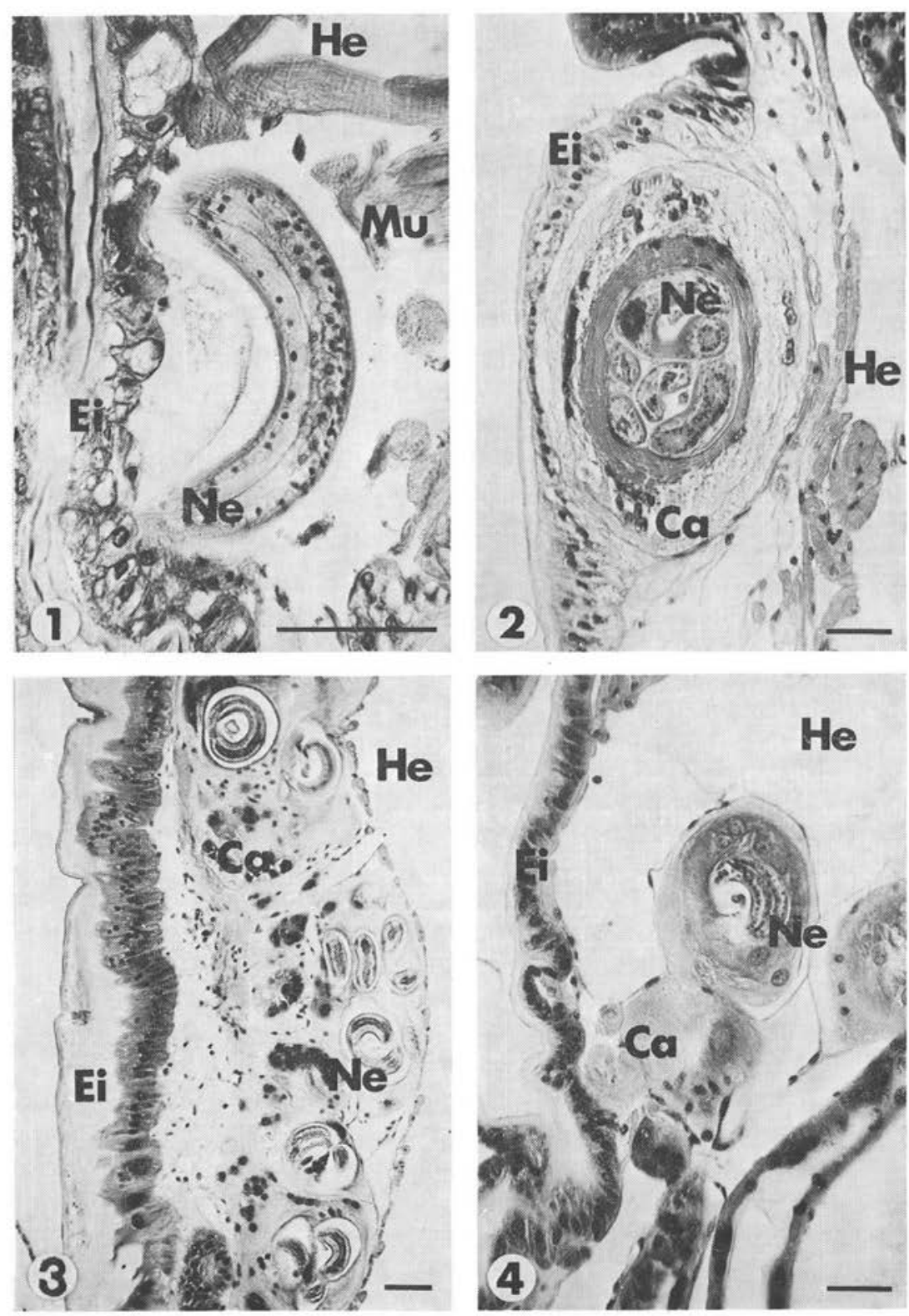
- Intérêt taxonomique. Avec l'espèce $P$. affinis, la morphologie céphalique du troisième stade larvaire des Rictulariidae est actuellement connue chez les espèces P. zygodontomis (Quentin, 1967), P. hispanica Quentin, 1973, P. desportesi (Chabaud et Rousselot, 1956), P. taterilli (Baylis, 1928), Rictularia proni Seurat, 1915.

A l'exception des deux espèces $P$. zygodontomis et $P$. hispanica, qui ont une morphologie primitive et dont les structures céphaliques des troisièmes stades larvaires sont semblables, les autres espèces, dont les caractères adultes sont plus évolués, présentent déjà au troisième stade larvaire des structures céphaliques différentes, dont les particularités semblent spécifiques; au demeurant, il n'est pas étonnant de retrouver, chez le troisième stade larvaire de $P$. affinis, dont l'adulte est hautement différencié, les structures céphaliques larvaires les plus complexes.

De ce fait, nous n'hésitons pas à identifier à $P$. affinis la larve de Spiruride décrite par Chabaud (1954) sous le nom d'Agamospirura sp. $\mathrm{n}^{\circ} 3$ et récoltée au Maroc chez Akis elegans Charp., car cette larve de Rictulaire, dont la localisation particulière * à la limite entre l'intestin moyen et l'intestin postérieur» avait déjà été remarquée par l'auteur, est identique, à la fois par sa morphologie générale et par ses structures céphaliques, à nos spécimens.

- Ecologie et répartition biogéographique du parasite. Hormis les deux cas d'infestation spontanée que nous venons de signaler chez les Pimélies d'Afrique du Nord Tachyderma hispida et Akis elegans, des formes infestantes de $P$. affinis ont été récoltées dans le mésentère ou dans la paroi infestante de Reptiles par Witenberg (1928) et par Gupta et Pande (1970).

L'écologie du cycle de ce Nématode Rictulaire semble présenter une grande souplesse, car le Carnivore, hôte définitif, peut s'infester soit en ingérant directement les Insectes hôtes intermédiaires, soit en mangeant de petits Reptiles hôtes paraténiques.

Le développement du parasite est donc parfaitement adapté dans les conditions naturelles à l'éthologie du Carnivore hôte définitif, et explique ainsi sa très large répartition géographique.

La synonymie de $P$. cahirensis (Jägerskiold, 1904) et de $P$. splendida (Hall, 1913) avec $P$. affinis démontrée par Gibbs, 1957 permet de regrouper dans le spectre d'hôtes de ce Rictulaire principalement des Canidae (Renard, Chacal, Coyote, Chien domestique), mais aussi des Felidae (Chat domestique), Mustelidae (Lutra lutra L.) et des Viverridae (Viverricula indica Desmaret). La répartition géographique du parasite s'étend à toute l'Afrique, Madagascar, les Amériques, l'Asie ; il est signalé également dans le Sud de l'U.R.S.S., dans la région de Samarkand, par Irgashev (1958-1965), en Hongrie par Kobulej (1951) et en Espagne par Simon Vicente (1968).

- Etude comparée des réactions cellulaires provoquées chez l'Insecte hôte intermédiaire par $P$. affinis et par d'autres Rictulariidae. Une première comparaison entre l'encapsulement des larves infestantes de $P$. affinis chez l'hôte intermédiaire naturel (Tachyderma hispida, Coléoptère), d'une part, et, chez un hôte expérimental (Locusta 
migratoria, Orthoptère), d'autre part, montre que, dans le premier cas, le parasite ne provoque pas de réaction histopathologique sensible, alors que l'on peut en observer une dans le second cas. L'adaptation à l'hôte intermédiaire semble donc mieux réalisée chez la Pimélie, où le parasite est pratiquement en contact avec l'hémocèle, sans pour autant provoquer de réaction hémocytaire. Chez le Criquet, par contre, les larves sont isolées de l'hémocèle par une capsule épaisse. La localisation de l'encapsulement et le déroulement du développement jusqu'au stade infestant sont cependant identiques dans les deux cas.

L'étude comparée des capsules de $P$. affinis à celles de $P$. hispanica et de $P$. taterilli, chez le même hôte intermédiaire expérimental (Locusta) montre des différences sensibles dans la taille $(150$ à $200 \mu$ pour $P$. affinis, 250 à $300 \mu$ pour $P$. hispanica et $P$. taterilli), ainsi que dans le comportement de la capsule, qui est libérée dans l'hémocèle pour $P$. hispanica (Quentin et Seureau, 1974), alors que celles de $P$. taterilli et de $P$. affinis restent insérées dans la paroi du tube digestif ( $P l$. V, fig. 3 et 4$)$.

Les Nématodes Rictulaires forment un groupe de Spirurides très diversifié, où il est possible de classer les espèces à leur place zoologique respective, selon leur degré de différenciation morphologique (Cf. Quentin, 1969).

L'étude comparée des réactions celiulaires provoquées, dans des conditions expérimentales identiques, par trois espèces $P$. (Paucipectines) hispanica, P. (Mesopectines) taterilli et $P$. (Multipectines) affinis, situées à trois étapes différentes de l'évolution morphologique du groupe (primitive, moyennement différenciée, très différenciée), montre que la réaction du tissu parasité de l'Insecte-hôte intermédiaire présente également des différences.

Cependant, dans l'état actuel des observations réalisées, il est encore aléatoire d'interpréter ces différences en degrés d'adaptation et de les rapporter aux données morphologiques des larves, afin d'établir plus sûrement la sériation taxonomique de Nématodes au sein d'un même genre, alors que cela peut être proposé pour des Nématodes appartenant à des familles différentes ou observé entre genres d'une même famille.

\section{Bibliographie}

Chabaud (A.-G.), 1954. - Sur le cycle évolutif des Spirurides et de Nématodes ayant une biologie comparable. Valeur systématique des caractères biologiques. Ann. Parasit. hum. comp., 29, 42-88, 206-249, 358-425.

GibBs (C. H.), 1957. - The taxonomic status of Rictularia affinis Jägersk. 1909, R. calurensis Jägersk. 1909, and R. splendida Hall, 1913. Canad. J. Zool., 35, 405-410.

GuPta (U. P.) et PANDE (B. P.), 1970. - Hemidactylus flaviridis, a paratenic host of Rictularia calurensis. Curr. Sci., 39, 535-536.

IRGASHEV (I. K.), 1958. - L'Helminthofaure des Carnivores domestiques et sauvages dans la région de Samarkand. Uzbek. biol. Zh., 5, 39-45.

IRGASHEv (I.-K.), 1965. - Helminthofaune des chats domestiques et sauvages dans la région de Samarkand. Trudy uzbek. nauchno. issled. Inst. vet., 17, 99-101. 
KobuleJv (T.), 1951. - On the incidence of Rictularia affinis Jägerskiold 1904 in the Hungarian red fox, with a redescription of this species. Acta Veterinaria Academiae Scientiarum Hungaricae Budapest, 1, 394-404.

Quentin (J.-C.), 1969. - Essai de classification des Nématodes Rictulaires. Mém. Mus. nat. Hist. nat., nlle ser. ser. A, Zool., 54, 55-115.

Quentin (J.-C.), 1970. - Cycle biologique de Pterygodermatites (Mesopectines) taterilli (Baylis, 1928) Nematoda Rictulariidae. Ann. Parasit. hum. comp., 45, 629-635.

Quentin (J.-C.) et Seureau (C.), 1974. - Cycle biologique de Pterygodermatites hispanica Quentin, 1973 (Nematoda, Rictulariidae). Ann. Parasit. hum. comp., 49, 701-719.

Seureau (C.), 1973. - Réactions cellulaires provoquées par les Nématodes Subulures et Spirurides chez Locusta migratoria (Orthoptère) : localisation et structure des capsules. Z. Parasitenk., 41, 119-138.

Simon Vicente (F.), 1968. - Los Rictularia (Nematoda: Spiruroidea) de Vulpes sp. en dos provincias de España. Rev. iber. Parasit., 28, 1-18.

Witenberg (G.), 1928. - Reptilien als Zwischenwirte parasitischer Würmer von Katze und Hund. Tierärztliche Rundschau, 34, 603-604. 